\title{
脂代谢紊乱在非酒精性脂肪肝 发病中的作用
}

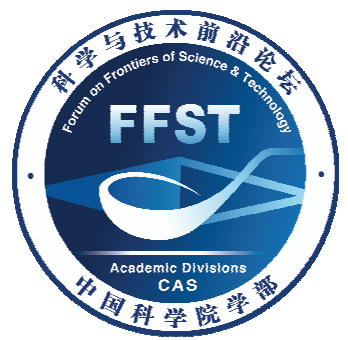

\author{
㫿玉兰，韩泽广* \\ 上海交通大学系统生物医学研究院, 上海 200240 \\ * 联系人, E-mail: hanzg@sjtu.edu.cn
}

收稿日期: 2017-04-10; 接受日期: 2017-05-10; 网络版发表日期: 2017-05-19

\begin{abstract}
摘要随着肥胖、2 型糖尿病、代谢综合征等疾病发病率的增加, 非酒精性脂肪性肝病(NAFLD)全球患病率也 逐年递增. 其发病机制较为复杂, 通常伴随着胰岛素抵抗、脂代谢紊乱、脂毒性调亡、炎症反应失调等现象. 本 文着重对脂代谢紊乱在 NAFLD 中的作用及其机制进行了综述, 同时对细胞脂毒性、胰岛素抵抗及肠道菌群紊乱 在其中的作用也进行简要描述，旨在为 NAFLD 的预防和治疗提供新思路.
\end{abstract}

关键词 NAFLD, 脂质代谢

肝脏参与脂质代谢的多个重要环节，包括 FA(fatty acid, FA)的摄取与合成, 脂质的加工、贮存、 氧化分解及输出等. 过去, 甘油三酯 (triglyceride, $\mathrm{TG)}$ 的沉积被认为是造成细胞损伤的“元凶”, 现在越 来越多的研究表明 FA 及其相关代谢产物才是造成脂 毒性的关键, 而细胞内脂质累积及 $\mathrm{TG}$ 还能保护细胞 免受损伤 ${ }^{[1]}$. 目前, FA 及其相关代谢产物成为脂代谢 研究的重点. 肝脏 FA 的来源主要有: (i) 外周脂肪组织 释放出非酯化游离 FA(nonesterified fatty acid, NEFA), 沉积到肝脏; (ii) 肝脏 FA 的从头合成途径 (de novo lipogenesis, DNL); (iii)食物中 FA 通过血浆 NEFA 或者 肠道乳糜颗粒形式的吸收进入肝脏. 近年来, 饥饿状 态下的自噬途径也被认为可以通过脂 滴降解, 将 $\mathrm{FA}$ 释放进入细胞质. 而肝脏 $\mathrm{FA}$ 利用主要指脂质氧 化和与蛋白质结合形成脂蛋白分泌出肝脏(图 1).
非酒精性脂肪性肝病 (nonalcoholic fatty liver disease, NAFLD) 以肝细胞的脂肪变性和脂肪贮积为 病理特征, 并排除饮酒、药物、遗传疾病等因素. 目 前认为, NAFLD 主要有三个阶段, 即单纯性脂肪肝 (nonalcoholic simple fatty liver, NAFL)、脂肪性肝炎 (nonalcoholic steatohepatitis, NASH)和肝纤维化阶段. 有些患者还会进展为肝硬化甚至肝细胞癌. 临床上, NAFLD 患者经常伴有肥胖、糖尿病或代谢综合症. 以往, Day 等 ${ }^{[2,3]}$ 曾提出 “二次打击学说”, 认为代谢紊 乱导致的肝细胞内 TG 沉积是 NAFLD 发展的第一重 打击, 随后毒性脂代谢产物累积和活性氧化物质 (reactive oxygen species, ROS)产生过度, 引起细胞调 亡及炎症反应异常活化, 成为第二重打击, 最终进展 为 NASH. 近年, 随着“多次打击学说”被提出, 开始 强调内质网应激、肠源性内毒素、脂肪因子失衡、肝

引用格式: 翟玉兰, 韩泽广. 脂代谢紊乱在非酒精性脂肪肝发病中的作用. 中国科学: 生命科学, 2017, 47: 496-507

Qu Y L, Han, Z G. Hepatic lipid metabolism and non-alcoholic fatty liver disease. Sci Sin Vitae, 2017, 47: 496-507, doi: 10.1360/N052017-00103 


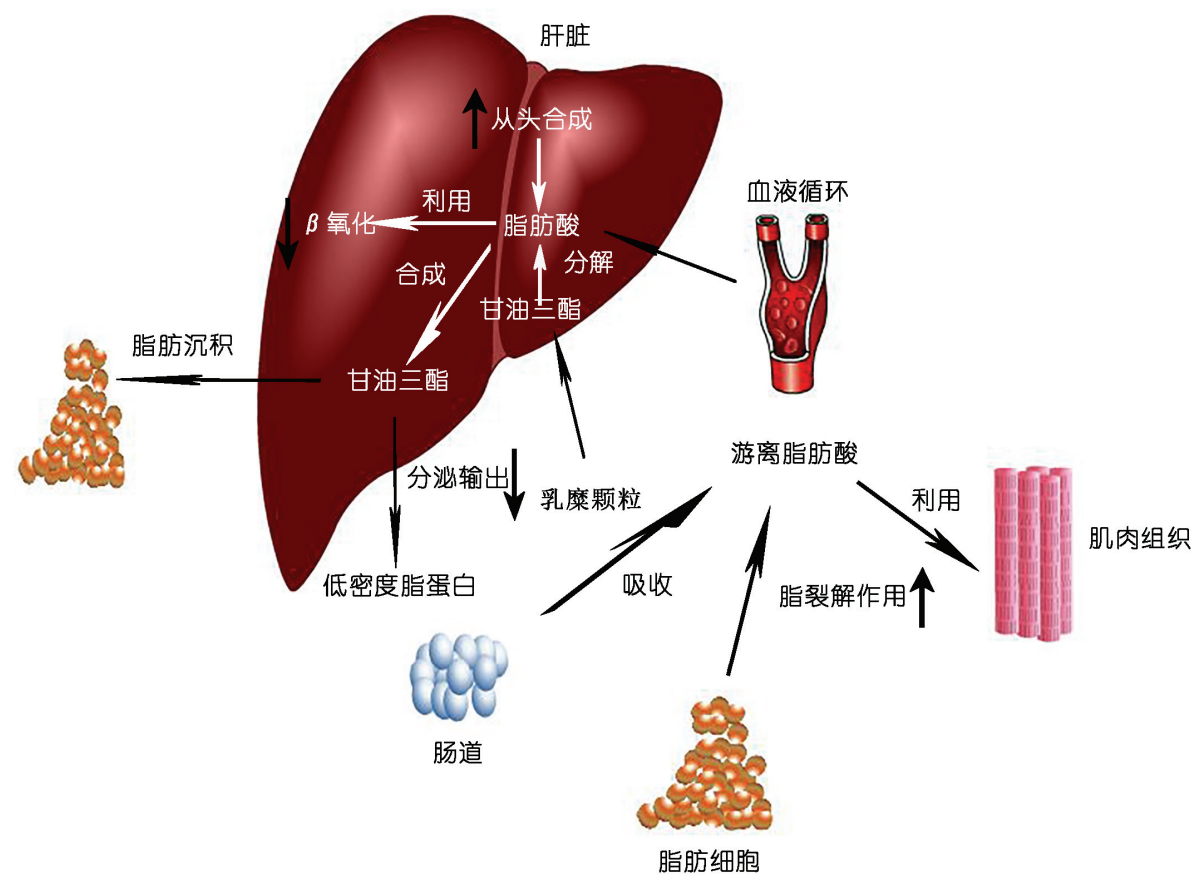

图 1 肝脏 FA 的来源与去处 (网络版彩图)

NAFLD 病人往往伴有肥胖、 2 型糖尿病或胰岛素抵抗, 其外周脂肪组织脂裂解率增强, 肝脏从头合成途径也显著提高, 但其 FA 的氧化及输

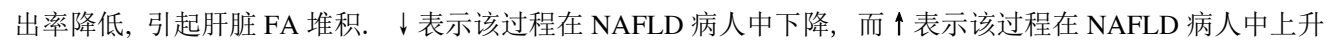

星形细胞激活等在 NAFLD 发生发展中的重要作用. 本文主要对 NAFLD 发病过程的脂质代谢重要环节异 常及其相关调控因子综述与探讨, 旨在为 NAFLD 的 预防和治疗提供新思路.

\section{1 肝脏脂质堆积的发生机制}

\section{1 食物中脂肪量增加}

在进入血液前, 食物中的脂肪须在小肠肠腔进 行乳化和水解. 正常情况下, 肝脏合成胆汁酸并经胆 管分泌至肠腔将脂肪乳化成乳糜颗粒, 与脂肪水解 酶类充分接触并水解, 水解后的脂肪被小肠上皮细 胞吸收. 乳糜颗粒在进食两小时后进入血液循环, 其 可结合载脂蛋白 apoE 和 apoC-2, apoC-2 进一步激活 脂肪酶(lipoprotein lipase, LPL), 将脂肪水解成甘油 和 $\mathrm{FA}^{[4]}$. 部分 $\mathrm{FA}$ 可被脂肪细胞吸收并存储, 剩余部 分会进入血液循环. 肝脏低密度脂蛋白(low-density lipoprotein, LDL)受体和其他相关蛋白识别 apoE, 进 一步吸收剩余颗粒, 随后经细胞内的溶酶体加工处 理. 若食物中脂肪含量过高, 就会引起血中 FA 含量
显著增加, 引起肝脏脂肪变性.

\section{2 外周组织 FA 摄取量增强}

肝脏还可从血浆中获得 NEFA, 其主要源自于脂 肪细胞的脂裂解作用. 这种情况通常发生在饥饿状 态下, 这个过程受到胰岛素的抑制. 研究表明, 在肥 胖及代谢综合症等存在胰岛素抵抗的状况下，体内 的脂裂解作用明显增强, 引起血中 NEFA 含量的显著 增加，可直接增强肝脏对 NEFA 的摄取 ${ }^{[5]}$.

肝脏摄取 NEFA 不是简单的被动扩散，而是有 多种膜转运蛋白介导参与. 肝细胞表达的 FA 转运蛋 白包括小凹蛋白(caveolins)、FA 转运蛋白(fatty acid transport protein, FATPs)、FA 转位酶 (fatty acid translocase, FAT/CD36)及 FA 结合蛋白(fatty acid binding protein, FABPs)等.

\subsubsection{Caveolins}

脂筏是细胞膜上一段富含鞘脂和胆固醇的结构, 研究发现，这段结构与脂质的吸收密切相关. 细胞膜 上脂筏与小凹蛋白作用后内陷形成囊泡, 将膜蛋白 和其他组分募集在一起参与物质转运和细胞信号转 
导. 小凹蛋白家族包括三个成员, 即 caveolin-1, caveolin-2 和 caveolin-3. 多数细胞都表达 caveolin-1 和 caveolin-2, 而 caveolin-3 主要在心肌和骨骼肌细胞表 达. 研究发现, 内皮细胞膜小凹上有大量信号转导分 子, 如受体酪氨酸激酶受体, $\mathrm{G}$ 蛋白偶联受体, 转化 生长因子 $\beta$ 受体等, 它们在蛋白包裹, 脂滴形成和信 号转导方面发挥了重要作用 ${ }^{[6]}$. Caveolin-1 缺陷小鼠 (Mus musculus) 可以抵抗高脂饮食诱导的肥胖 ${ }^{[7]}$; 而 肝脏特异性 caveolin-1 缺陷小鼠的肝脏脂质沉积现象 也明显低于对照组 ${ }^{[8]}$.

\subsubsection{FATPs}

FATPs 家族含有 6 个成员, 其中 FATP1 和 FATP6 分别主要在肌肉, 脂肪组织和心脏中表达, FATP4 在小肠和脂肪组织中含量较多, 而 FATP2 和 FATP5 在肝脏中表达丰富, 在 FA 吸收过程中发挥着 重要作用.

FATPs 的 RNA 转录和蛋白表达受到激素、炎症 因子、转录因子调控, 对 FA 稳态维持不可或缺. 在 6 个成员中, FATP4 与人类肥胖和胰岛素抵抗相关, 而 FATP5 与肝脏脂代谢密切关联. 实验表明, FATP5 过 表达可以显著增加 FA 的吸收; 而肝脏特异性 FATP5 缺失的小鼠肝脏 FA 吸收量相对于野生型对照组下降 了 $50 \%$, 即使在高脂饮食下, 缺陷型小鼠肝脏 TG 的 累积也明显减少, 胰岛素敏感性增加 ${ }^{[9]}$, 提示肝脏 FATP5 在 FA 吸收过程中的发挥重要作用.

\subsubsection{FAT/CD36}

FAT/CD36 蛋白是一个约为 $80 \mathrm{kD}$ 大小的细胞膜 糖蛋白, 属于清道夫受体家族成员, 在多种组织、细 胞中广泛表达, 可与多种配体结合, 行使不同的细胞 功能. 在 FA 代谢过程中, 其作用主要是从脂蛋白中 解除并游离出 $\mathrm{FA}$, 并催化质子化的 $\mathrm{FA}$ 整合进入细 胞膜的外层磷脂层中.

与其他转运蛋白相比, FAT/CD36 没有组织特异 性, 除了增加 FA 的吸收, FAT/CD36 还可以与胶原蛋 白、极低密度脂蛋白(very low-density lipoprotein, VLDL)等蛋白结合. 与野生型对照组相比, FAT/ $\mathrm{CD} 36$ 基因敲除小鼠肌肉的胰岛素敏感性增强、脂肪 和肌肉组织中 $\mathrm{TG}$ 含量下降, 但是血浆游离 $\mathrm{FA}$ 和肝 脏 TG 含量增加, 同时肝脏胰岛素敏感性降低 ${ }^{[10]}$, 这 可能由于 FAT/CD36 缺陷导致外周游离FA吸收减少,
血中 FA 增多, 使得更多 FA 进入肝脏. 在正常情况 下, FAT/CD36 的表达很低, 主要由其他蛋白在发挥 作用. 但在特殊条件下, 如高脂饮食、糖尿病、肥胖, FAT/CD36 就会影响脂肪肝的发展 ${ }^{[11,12]}$. 实验表明, 过表达 FAT/CD36 的小鼠体内 TG 含量显著增加, 而 降低 FAT/CD36 表达可以减缓脂肪肝的发生 ${ }^{[13]}$. 在 NAFLD 患者肝脏中, CD36 的表达增加且与脂滴含量 呈现正相关性 ${ }^{[14]}$.

\subsubsection{FABPs}

FABP 家族是一组协调脂质运输和反应的细胞 内蛋白质, 可直接转运 FA 代谢产物、参与细胞内信 号传递过程. 该家族有 9 个成员(FABP1-9), 其中 FABP4 研究最多, 它主要在脂肪细胞和巨噬细胞中 高表达, 可以被长链 FA、过氧化物酶体增殖物激活 受体 (peroxisome proliferator activated receptors, PPARs)及 Toll 样受体(toll-like receptors, TLRs)激动 剂等活化, 进而参与糖、脂代谢及炎症反应过程 ${ }^{[15-17]}$. FABP4 结合长链FA, 并携其穿梭于细胞膜、细胞器 和其他蛋白之间, 影响 FA 的摄取、运载、酯化和 $\beta$ 氧化等环节, 从而对 FA 氧化及磷脂、 $\mathrm{TG}$ 代谢起调节 作用; FABP4 还活化脂肪细胞的激素敏感脂肪酶, 调控脂解作用. 此外, FABP4 还与细胞内蛋白络氨酸 激酶 JAK 结合而减弱其信号, 从而影响脂质代谢.

FABP4 缺陷型小鼠能够抵抗高脂饮食诱导的胰 岛素抵抗及高血糖症. 与动物结果一致的是, 人群中 FABP4 基因位点突变导致其表达量减少, 降低了 2 型 糖尿病的发病率. 前瞻性研究还发现高水平 FABP4 是代谢综合征及 2 型糖尿病发病的独立预测因 子 ${ }^{[18,19]}$, 这些结果提示其在代谢疾病发生过程中发 挥重要作用.

\subsection{DNL 途径}

肝脏脂肪的 DNL 途径可将体内多余的糖类转化 为 $F A$, 其受激素及中间产物调控. 乙酰辅酶 $\mathrm{A}$ 先后 在乙酰辅酶 $\mathrm{A}$ 羧化酶 (acetyl CoA cal-boxylase, $\mathrm{ACC} 1$ )、FA 合成酶(fatty acid synthase, FAS)及硬脂酰 辅酶 A 去饱和酶(stearoyl-CoA desaturase, SCD)作用 下生成脂酰辅酶 $\mathrm{A}$, 其与磷酸甘油一起在甘油-3-磷 酸酰基转移酶 (glycerol-3-phosphate acyl-transferase, GPAT)和(diacylglycerol acyltransferase, DGAT)作用 下生成 TG(图 2). 值得注意的是, 上述这些编码酶类 


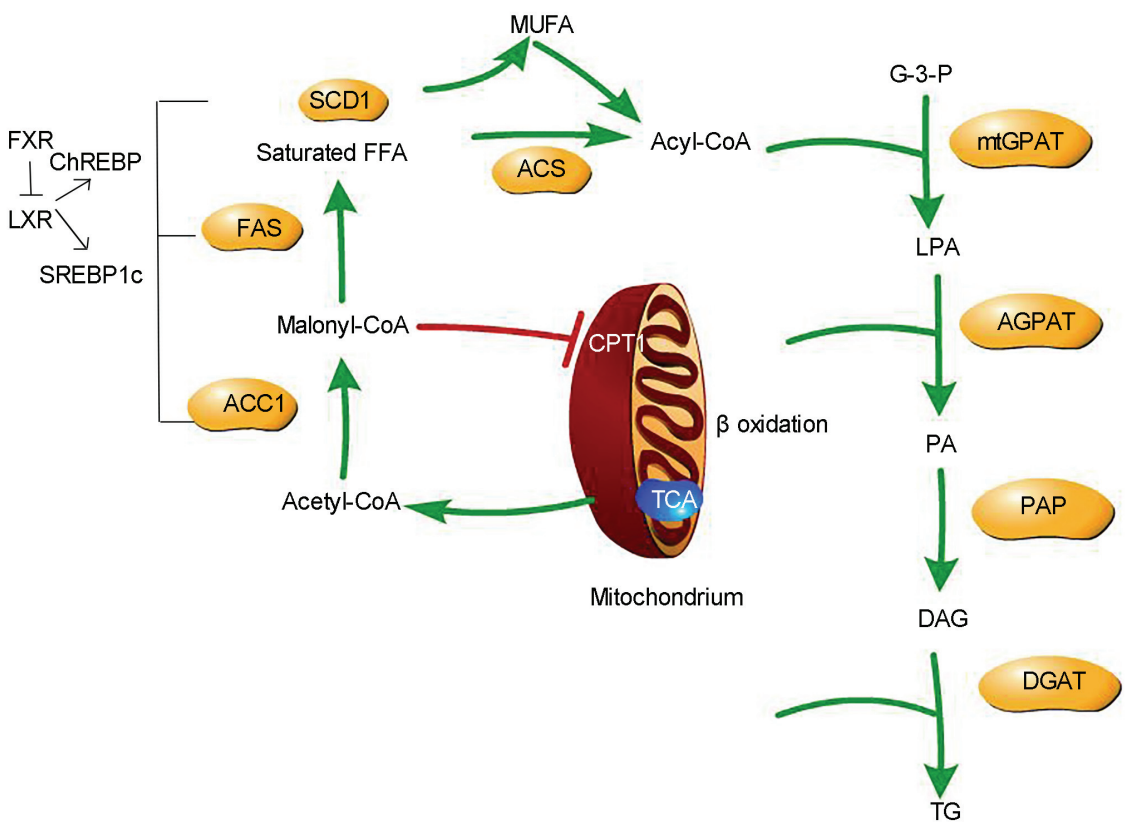

图 2 肝脏 TG 的合成途径及调控该过程的转录因子 (网络版彩图)

Acetyl-CoA: 乙酰辅酶 A; Malonyl-CoA:丙二酰辅酶 A; Saturated FFA: 饱和 FA; MUFA:单不饱和 FA; Acyl-CoA:辅酶 A 酯; G-3-P:3-磷酸葡萄 糖; LPA:溶血磷脂酸; PA: 磷脂酸; DAG: 甘油二酯; TG: TG. ACC1: 乙酰辅酶 A 羧化酶 1; FAS: FA 合成酶; SCD1: 硬脂酰辅酶 A1; ACS: 酯 酰辅酶 A 合成酶; GPAT: 甘油-3-磷酸酰基转移酶; AGPAT: 溶血磷脂酸酰基转移酶; PAP: 磷脂酸磷酸酶; DGAT: 二酰甘油酰基转移酶; CPT1: 肉毒碱棕榈酰转移酶; TCA: 三羧酸循环

基因的表达水平受一些代谢相关的转录因子调控, 包括固醇调节元件结合蛋白(sterol-regulatory element binding protein, SREBP)、碳水化合物反应元件结合蛋 白 (carbohydrate response element binding protein, ChREBP)、肝 X 受体(liver X receptor, LXR)、法尼酯 $\mathrm{X}$ 受体(farnesoid $\mathrm{X}$ receptor, FXR)、PPARs 等.

\subsubsection{SREBPS}

SREBPs 家族主要含 SREBP1a，SREBP1c 和 SREBP2 三个成员. SREBP1a 和 SREBP1c 由同一个基 因编码, 其中 SREBP1c 主要在小鼠和人肝脏、白色 脂肪组织、肾上腺和大脑中表达, 而 SREBP1a 在脾 脏、肠道等组织高表达. SREBP2 是由另外一个基因 编码，其与 SREBP1 约 50\%同源性.

SREBP1c 参与肝脏脂质合成过程. 过表达 SREBP1c 可引起肝脏脂质堆积和胰岛素抵抗 ${ }^{[20]}$; 在 伴有肝脏脂质变性的肥胖小鼠体内, SREBP1c 表达显 著增加 ${ }^{[21]}$. 然而, SREBP1c 缺陷小鼠在喂食后不能有 效活化脂质合成途径中的关键酶 ACC1, FAS, SCD1 等. 但是 SREBP1c 缺陷只能抑制约 50\%的脂质合成
相关基因表达，提示脂质合成不完全依赖于 SREBP1 $c^{[22]}$.

SREBP1c 还参与胰岛素信号途径. 胰岛素通过 AKT 和 mTOR 两个途径活化 SREBP1c, 其中 AKT 可以直接磷酸化 SREBP1c, 增强其转运至高尔基体 进行切割加工活化能力 ${ }^{[23,24]}$; 而 mTOR 和其下游 S6 分子也可以促进 SREBP1c 的加工处理 ${ }^{[25,26]}$. SREBP1c 还可以被 MAPK 分子如 $\mathrm{p} 38$, ERK 和 JNK 磷酸化, 调控其结合 DNA 能力和转录活性, 从而影 响脂质合成相关基因表达.

此外，代谢途径还可以通过翻译后调控机制影 响 SREBP1c 分子功能. 在高糖和胰岛素刺激下, SREBP1c 的 K289 和 K309 分别被 p300/CBP 和 sirt3 乙酰化和去乙酰化，进而影响其调节脂质合成相关 基因表达作用 ${ }^{[21]}$.

\subsubsection{ChREBP}

ChREBP 蛋白由 864 个氨基酸构成, 含有 bHLH-Zip 功能结构域, 与另一个含 bZIP 结构的 MLX (Max-like protein X)蛋白形成异聚体,再结合碳 
水化合物反应元件(ChoRE)进一步调节靶基因. 葡萄 糖升高可直接增强 ChREBP 的表达和转录活性, 也 可通过 LXR 间接上调 ChREBP 的表达, 促进糖酵解 相关酶如 L-PK, G6PC, GLUT4, GPDH 和 GKRP79 表 达 ${ }^{[27 ~ 31]}$. ChREBP 对脂肪合成相关基因如 ACLY, FAS, ACC 和 SCD18 表达也有调控作用 ${ }^{[32,33]}$.

ChREBP 基因缺陷小鼠糖酵解作用减弱, 脂生成 能力下降了约 $60 \%$, 同时伴随有胰岛素抵抗 ${ }^{[33]}$. 肝脏 ChREBP 特异性敲除会加重小鼠肥胖、肝脏脂肪变性 和胰岛素抵抗现象 ${ }^{[34]}$. 而过表达 ChREBP 虽然可增 加肝脏 TG 的累积, 但可改善高脂饮食引起的胰岛素 抵抗 ${ }^{[35]}$. 虽然 ChREBP 可以调控脂质合成相关基因 的转录, 但是肝脂肪变性程度与胰岛素抵抗并不总 是呈现相关性.

\subsubsection{LXR}

$\mathrm{LXR}$ 有 $\mathrm{LXR} \alpha$ 和 $\mathrm{LXR} \beta$ 两个亚型, 其中 $\mathrm{LXR} \alpha$ 在 肝脏中表达丰富. 若小鼠同时敲除 $\mathrm{LXR} \alpha$ 和 LXR $\beta$, 其肝脏脂质合成能力下降 $80 \%$, 提示 LXR 在肝脏脂 质合成过程发挥重要作用 ${ }^{[36,37]}$. 研究表明, LXR $\alpha$ 可 以通过结合和活化 SREBP1c 调控脂质合成相关基因 的转录 ${ }^{[38,39]}$. ChREBP 启动子区域也存在 LXR $\alpha$ 的结 合位点, LXR $\alpha$ 激动剂可以增加 ChREBP 的表达 ${ }^{[40,41]}$, 但 LXR $\alpha$ 和 LXR $\beta$ 双敲除小鼠 ChREBP 表达没有变 化 ${ }^{[42]}$. 此外, FAS, ACC 和 SCD 这些脂质合成相关基 因启动子区域本身也有 $\mathrm{LXR} \alpha$ 结合位点 ${ }^{[43]}$, 所以 LXR 既可以直接调控又可以通过 SREBP 和 ChREBP 间接调控脂质合成过程.

\subsubsection{FXR}

FXR 也参与到糖脂代谢调控过程中, 可以通过 结合 PGC1 $\alpha$, 增加糖生成量、促进 FA $\beta$ 氧化、抑制 脂质合成基因表达 ${ }^{[44]}$. FXR 可协同核受体 SHP(small heterodimer partner)抑制 LXR 的表达, 后者调控 SREBP1c 从而影响脂质合成过程 ${ }^{[45}$. FXR 还通过上 调 apoC2, apoC3 和 VLDL 受体加速清除富含 TG 的 脂蛋白. 体内实验进一步证实 FXR 的作用, FXR 缺陷 小鼠在高胆固醇和高脂饮食后, 出现了严重的肝纤 维化 ${ }^{[46]}$.

\subsubsection{PPARs}

PPARs 是配体激活的转录因子, 属于核受体超 家族成员, 包括 PPAR $\alpha, \operatorname{PPAR} \gamma, \operatorname{PPAR} \delta$ 三种亚型.
其中 PPAR $\alpha$ 是调节脂质代谢的最关键因子, 在代谢 活性较高的组织如肝脏、肌肉、脂肪组织等高表达. 在脂代谢方面,PPAR $\alpha$ 可调节 FA $\beta$ 氧化、FA 运输等 过程 ${ }^{[47]}$. PPAR $\alpha$ 激动剂能够降低血浆 TG 和 LDL 水 平, 升高高密度脂蛋白(high density lipoprotein, HDL) 水平, 从而改善脂质代谢 ${ }^{[48]}$.

PPAR $\alpha$ 促进肝细胞 FA 的氧化, 包括上调 $\beta$ 氧化 过程中的关键酶、增强 FA 在线粒体和过氧化物酶体 中的氧化能力, 还可以增加 CYP2E1 和 CYP4A11 的 表达, 增强内质网 $\omega$ 氧化 ${ }^{[49]}$. 此外, PPAR $\alpha$ 增加肝脏 ApoA1 的表达, 促进 HDL 成熟和代谢. 而 PPAR $\gamma$ 在 脂肪细胞表达较高, 激活后与 RXR 形成异二聚体, 结合到靶基因的 PPAR 反应元件(PPAR response element, PPRE), 抑制 FA 分解和释放相关基因表达, 同时增加 FA 储存相关基因表达，从而降低小鼠的血 浆 $\mathrm{TG}$ 和游离 $\mathrm{FA}$ 水平、抑制炎症因子的产生 ${ }^{[50]}$.

\section{4 经溶酶体途径产生 FA 增多}

近年来，自噬将 $\mathrm{TG}$ 残余物降解、释放出 $\mathrm{FA}$ 的 过程被认为在脂代谢过程中也发挥了一定作用. 自 噬作为溶酶体降解途径, 在饥饿状态下, 可以通过回 收利用为一些重要供能途径提供重要成分 ${ }^{[51]}$, 在该 过程, 脂滴被溶酶体包裹形成自噬小体后被降解、释 放出游离 FA 参与 $\beta$ 氧化 ${ }^{[52]}$. 饥饿会抑制 mTOR 信号 途径，后者可以抑制自噬. 持续抑制自噬会影响胰岛 素敏感性和 AKT 介导的 FOXO 磷酸化, 后者是自噬 相关基因 ATG 的活化因子 ${ }^{[53]}$. 但胰岛素抵抗或肥胖 患者肝脏 mTOR 呈现过度活化, 而 ATG 抑制因子 calpain 表达增加 ${ }^{[54]}$.

\subsection{FA 氧化途径失调}

在肝细胞内, FA 氧化可发生在线粒体、过氧化物 酶体及内质网, 是一种非常有效且快速的产能方式. 短链和中链 FA 不需要活化即可直接透过线粒体膜, 而长链 FA 在参加代谢前需经脂酰 CoA 合成酶(acyl CoA synthetase, ACS)活化, 再经肉碱棕榈酰转移酶 (camitine palmitoyl transterase-1, CPT1) 转运才能进 入线粒体进行 $\beta$ 氧化, 而超长链 FA 则被转运至过氧 化物酶体进行 $\beta$ 氧化 ${ }^{[55]}$. 丙二酰辅酶 $\mathrm{A}$ (malonyl-CoA) 作为 FA DNL 早期的中间体, 在胰岛素受体活化时在 细胞内累积, 抑制 CPT1 的活性 ${ }^{[56]}$. 在饥饿状态下, 细胞内 FA 氧化过程是受到抑制, 相反 FA DNL 活化, 
以确保脂类的存储和分布. 当细胞内 FA 过剩时, 长 链 FA 可在内质网中经细胞色素 P450 (cytochrome P450, CYP4A) 依赖性的 $\omega$ 氧化进行分解. FA 经两种 途径氧化分解会产生较多的 ROS 和脂质过氧化物. 如果 FA 量超过线粒体 $\beta$ 氧化处理能力, 则可造成 FA 毒性代谢产物在线粒体内蓄积, 从而导致线粒体功 能紊乱. 同时, 过氧化物酶体和内质网对 FA 的代谢 增强, ROS 和脂质过氧化物生成增加, 后两者可直接 造成细胞损伤, 甚至通过激活炎性反应, 导致 NASH 和肝纤维化(图 3).

在 NASH 病例中, 过氧化物酶体内的 $\beta$ 氧化和内 质网的 $\omega$ 氧化明显增加, 导致脂毒性和 ROS 的大量产 生, 这可能是线粒体 CPT1 酶活性受到抑制、 $\beta$ 氧化能 力降低造成的. 提高线粒体 $\beta$ 氧化可以缓解 NASH 和 胰岛素抵抗.

\section{6 脂蛋白输出和分泌过程受阻}

$\mathrm{FA}$ 会以 $\mathrm{TG}$ 的形式储存, 或以脂滴形式储存在 肝细胞中, 或进一步与 ApoB100 结合加工成 VLDL 分泌出去, 供周围组织摄取和利用. 在肝细胞利用 TG 合成 VLDL 分泌囊泡的过程中, ApoB 起到至关重 要的作用. ApoB 在内质网膜上的定位, 可启动 VLDL 囊泡组装过程, 并通过与 TG 转运蛋白(triglyceride transfer protein, MTP) 相互作用, 连同装配 ApoC3, ApoE 等环节, 促使分泌囊泡成熟. 生理状态下, 肝 细胞内 $\mathrm{FA}$ 和 $\mathrm{TG}$ 水平升高可刺激 $\mathrm{ApoB}$ 表达; 而在 胰岛素水平升高时, 则抑制肝脏 ApoB 表达, 从而抑 制 VLDL 输出. 当非酒精性脂肪肝伴 FA 显著升高和 胰岛素抵抗发生时, ApoB 表达和 VLDL 输出均增加, 但肝脏 VLDL 输出总量增加常常不能代偿肝脏脂肪

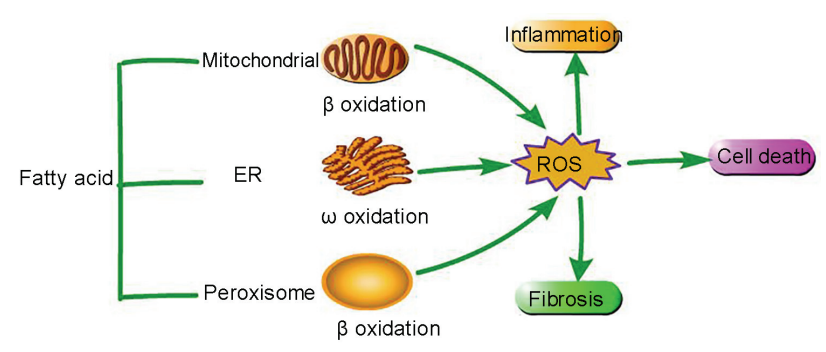

图 3 肝脏 FA 的氧化途径及效应 (网络版彩图)

Fatty acid: FA; PS: 磷脂酰胆碱; Mitochondiria: 线粒体; ER: 内质网; ROS:活性氧; Fibrosis: 纤维化; Inflammation: 炎症; Cell death: 细 胞死亡; oxidation: 氧化
沉积, 并且游离 FA 升高可增强促炎因子的活性, 导 致慢性炎症反应, 加重胰岛素抵抗, 形成恶性循 环 ${ }^{[57]}$. 除胰岛素外, 代谢相关的转录因子如 SREBP1c, LXR, FXR, FOXO 和 ChREBP 也参与调控 肝脏 VLDL 合成与分泌过程中. FOX01 增加 VLDL 分 泌囊泡中 ApoC3 表达, 促进 VLDL 合成和运出肝脏 ${ }^{[58]}$; PPAR $\alpha$ 抑制 ApoC3, 增加循环 VLDL 分解 ${ }^{[59]}$; FXR 可 抑制 VLDL 装配与分泌 ${ }^{[60]}$.

\section{2 脂毒性与细胞调亡和炎症反应}

当肝脏中游离 FA 及相关代谢产物增加超过其负 荷能力时, 会诱导大量的 ROS 产生, 产生氧应激, 此 外, 具有脂毒性的中间产物如神经酰胺, 二脂酰甘油 (DAG), 磷脂酰胆碱等显著增多, 造成脂质过氧化损 伤, 引起细胞调亡及炎症反应异常活化.

\section{1 细胞脂毒性}

原来的脂毒性概念用来描述过量的 NEFA 导致 胰岛 $\beta$ 细胞内脂酰辅酶 $\mathrm{A}$ 增加, 进而增加神经酰胺浓 度, 后者可诱导一氧化氮产生增多, 引起细胞毒性并 加速 $\beta$ 细胞调亡过程. 后来细胞脂毒性用来广泛描述 由 FA 及其相关代谢物造成细胞损伤及死亡事件. 但 是由于 FA 代谢产物种类繁多, 包括短链、长链、超 长链的区分, 饱和与不饱和的差异, 以及顺式与反式 的不同, 使得确切鉴定脂毒性分子类型变得具有挑 战性, 本文会列举一些目前研究较多的脂代谢产物.

\subsubsection{NEFA}

NEFA 通常与 FABPs 结合后被吸收的. 研究表 明, NASH 病人中, 虽然血浆中 NEFA 含量增加, 但 是肝脏中 NEFA 含量没有明显增加 ${ }^{[61]}$, 所以其含量 不是造成脂毒性的根本原因, 而是其被传递给相关 酶的能力提升了才是关键所在. 如果心肌细胞过表 达 FABPs 则会加重脂毒性 ${ }^{[62]}$, 相反, 肝脏 FABPs 缺 失, 则肝纤维化发生概率就会明显降低 ${ }^{[63,64]}$.

作为配体, NEFA 还可以结合 PPAR $\alpha, \operatorname{PPAR} \gamma$ 和 $\mathrm{G}$ 蛋白偶联受体, 间接参与这些蛋白介导的 FA 氧化 及存储过程中 ${ }^{[65]}$. 不饱和 NEFA 甚至还可以活化 TLR4 受体, 启动细胞内的调亡级联反应 ${ }^{[6,67]}$. FA 还 影响核激素受体途径, 后者可以进一步影响肝损伤 程度, 促进胰岛素抵抗和内质网应激 ${ }^{[65,68]}$, 导致细胞 
调亡和炎症反应.

\subsection{2 神经酰胺}

神经酰胺是另一种在细胞脂毒性中发挥重要 作用的 FA 代谢产物, 其在脂肪性肝病中含量显著增 加 ${ }^{[69]}$. 尽管有证据表明神经酰胺在胰岛细胞中有一 定的脂毒性 ${ }^{[70]}$, 但是在其他组织中阻断神经酰胺的 表达不能有效地减缓 FA 引起的损伤, 如用药物阻断 神经酰胺合成的最后一步, 但不能阻断由棕㭣酸诱 导的脂毒性肝细胞损伤 ${ }^{[71,72]}$. 在中国仓鼠卵巢细胞 中的实验表明, 虽然棕榈酸可以诱导神经酰胺含量 的增加, 但其并没有参与其引起的脂毒性过程中 ${ }^{[73]}$. 这些结果表明神经酰胺在棕榈酸诱导的脂毒性损伤 中效应不大.

\subsubsection{DAG}

DAG 可以活化大部分 PKC 激酶家族成员, 参与 多条信号通路调控中. 在 NAFLD 和 NASH 病人中, DAG 量也显著高于正常值 ${ }^{[61]}$. 研究发现, 在四氯化 碳 $\left(\mathrm{CCl}_{4}\right)$ 诱导的大鼠 (Rattus norvegicus) 肝损伤模型 中, $\mathrm{CCl}_{4}$ 可以诱导 $\mathrm{DAG}$ 过氧化物产生, 进而活化 $\mathrm{NF}-\kappa \mathrm{B}$ 信号通路及 $\mathrm{TNF} \alpha$ 介导的肝损伤 ${ }^{[27]}$.

\subsection{4 磷脂酰胆碱}

磷脂酰胆碱作为细胞膜的重要组成成分在 VLDL 的合成及分泌过程中起着重要作用. 研究表 明, 在 NAFLD 病人中, 磷脂酰胆碱含量显著增加, 且其含量与疾病的严重程度密切相关, 提示其在 FA 介导的毒性作用中的重要性 ${ }^{[71]}$. 小鼠研究也支持这 一观点, 实验发现, 磷脂酰胆碱引起的脂毒性肝细胞 损伤依赖于 $\mathrm{G}$ 蛋白偶联受体, 其主要通过引起线粒 体膜去极化而诱导细胞调亡所致.

\section{2 氧应激与脂质过氧化损伤}

当肝细胞中 NEFA 大量聚集时, 会促进线粒体 $\beta$ 氧化反应, 导致 ROS 增加, 当 ROS 产生超过抗氧化 系统清除能力时, 便会导致氧应激. 氧应激被认为是 肝脏脂肪变发展为脂肪性肝炎的催化剂, 主要通过 以下几个途径造成肝细胞损伤: (i)改变线粒体膜通透 性, 直接导致肝细胞死亡; (ii) 调节炎症应答相关细胞 因子和粘附因子如 TNF $\alpha, T G F \beta$ 表达; (iii)刺激花生四 烯酸代谢, 产生白三烯等类脂质趋化中性粒细胞; (iv)攻击生物膜上的不饱和 FA, 触发链式过氧化反应 产生脂质过氧化物, 后者激活肝枯否氏(Kupffer)细胞 和星状细胞, 引起炎症; 脂质过氧化物甚至可抑制抗 氧化剂活性,使细胞内 ATP 咜备和抗氧化物质减少, 形成活性氧、脂质过氧化及抗氧化能力下降之间的恶 性循环, 从而导致 NAFLD 和 NASH 所致的肝细胞变 性、坏死、炎症及纤维化发生.

\section{3 炎症}

Kupffer 细胞是肝脏内的巨噬细胞, 在 NAFLD 的发病中也起到重要的作用. 有研究认为, Kupffer 细 胞介导的免疫反应可能是 NAFLD 发病过程中造成肝 细胞损伤的基础. 大量的脂质堆积, 使 Kupffer 细胞 长期暴露于 “抗原”刺激下, 诱导产生 ROS, IL-6, C 反 应蛋白等表达, 引起持续性炎症反应 ${ }^{[74]}$. 也有研究显 示, 由 $\mathrm{T}$ 细胞产生的促炎因子增多、抗炎因子不足可 以影响脂肪性肝炎的形成; 活化的特殊 $\mathrm{T}$ 细胞亚 群-NKT 细胞可以直接损伤肝细胞 ${ }^{[75]}$. 此外, 脂肪组 织炎症也可影响肝脏病变. 在脂质堆积严重的肥胖 小鼠中, 脂肪组织的巨噬细胞分泌 $\mathrm{TNF} \alpha$ 显著升高, 从而增加脂肪细胞的脂裂解率, 促进肝脂肪变性. 小 鼠实验表明, 当过表达趋化因子 CCL2 时, 脂肪组织 内的巨噬细胞会被招募, 分泌大量炎症因子, 促进肝 脂肪变性; 而 CCL2 缺失可以抵抗高脂饮食诱导的脂 质堆积和胰岛素抵抗, 提示脂肪组织中巨喍细胞的 招募会调控脂质堆积.

\section{3 胰岛素抵抗与 NAFLD}

NAFLD 与 2 型糖尿病密切相关, 而肝脏所致的 胰岛素抵抗是两种疾病的共同点. 超过 $90 \%$ 的 2 型糖 尿病人患有 NAFLD, 其严重程度与 NAFLD 的病情 进展相关, 而 NAFLD 患者几乎都存在胰岛素抵抗的 现象, 但不一定伴有糖耐量异常或肥胖. 应用胰岛素 增敏剂可以改善肝脏的脂肪储堆积现象. 所以现在 有人认为胰岛素抵抗可能是第一次打击.

胰岛素除了通过上调葡萄糖转运蛋白含量促进 细胞对葡萄糖的吸收外, 还可促进脂类储存、抑制脂 类分解 ${ }^{[76]}$. 胰岛素抵抗与脂质聚集是一个相互促进、 相互影响的过程. 按逻辑推断, NAFLD 是肝脏疾病, 那么肝脏发生的胰岛素抵抗可能与 NAFLD 发病最相 关. 但研究表明, 肝脏特异性敲除胰岛素受体未引起 
NAFLD 或者脂毒性肝损伤; 而源于脂肪组织的胰岛 素抵抗对于脂毒性疾病包括 NAFLD 起到了重要的作 用, 源于脂肪细胞释放的 TNF 量增加, 促使脂肪 组织发生胰岛素抵抗, 进而增加脂裂解率. 然而, 肝 脏氧化作用抑制 NEFA 的利用, 从而导致脂肪在肝脏 细胞中的蓄积 ${ }^{[77]}$.

胰岛素抵抗与 NAFLD 的相关性在大鼠体内也得 到了证实, 当大鼠用高脂饮食处理三天后, 胰岛素抑 制肝脏糖生成能力受到了显著抑制, 并伴随着 DAG 含量和抑制胰岛素受体活性的 PKCE增多 ${ }^{[78]}$. 对伴有 胰岛素抵抗的肥胖患者的肝脏进行多种指标检测表 明, 与神经酰胺含量、炎症因子浓度和内质网压力等 指示相比, 肝脏 DAG 和 PKCE水平与胰岛素抵抗最 为相关 ${ }^{[79]}$.

\section{4 肠道菌群与 NAFLD}

前文提到, 肠道菌群紊乱在 NAFLD 的发生发展 及其脂代谢紊乱过程中的作用越来越受到重视. 肝 脏中 $70 \%$ 的血供是经门静脉从小肠获得的, 肝脏中 的肝细胞, 胆管上皮细胞和 Kupffer 细胞都表达天然 免疫受体, 会对肠道菌群来源的产物, 如肽聚糖, 内 毒素等做出反应 ${ }^{[00]}$. 了解肠道菌群紊乱, 肠道屏障完 整性和肝脏对肠道来源的因子的反应性对于提出新 的肝庄疾病治疗方法具有重要意义.

目前认为微生物菌群在 NAFLD 的发生发展上发 挥着多重作用, 包括改变微生物菌群结构, 提高内毒 素水平, 增加肠道屏障通透性, 改变胆碱代谢方式 等. 肠道菌群基因组测序结果提示 NAFLD 病人体内 肠道菌群数量与种类与正常个体存在差异, 其中菌 种 Bacteroidetes 和 Ruminococcaceae 的菌群数量明显
低于健康个体 ${ }^{[81,82]}$, 而另一菌种 Proteobacteria 在 $\mathrm{NASH}$ 病人中菌群数量明显增加 ${ }^{[83]}$. 最新的研究表 明, 如果在无菌小鼠体内移植高脂饲料喂养后罹患 高血糖小鼠的肠道菌群, 移植小鼠肝脏会呈现脂肪 变, 胰岛素抵抗等现象, 这一研究结果直接表明了肠 道菌群特征是可以传送的, 会直接影响 NAFLD 的发 生发展过程. 不仅如此, 多个小鼠模型证明菌群还会 影响血清中内毒素的水平, 内毒素是革兰氏阴性菌 的主要成分之一, 会通过活化肝脏 Kupffer 细胞来促 进炎症，进一步影响 NAFLD 的发病过程.

鉴于肠道菌群在 NAFLD 疾病中的作用, 越来越 多的研究开始以肠道菌群作为治疗该疾病的靶点, 方法主要包括补充肠道益生菌, 益生元等. 如外源性 的补给 Bifidobacterium, 可显著降低高脂饲料喂养后 罹患 NAFLD 的小鼠的肝脏 TG 积累量 ${ }^{[84]}$. 而 Fontana 等人的研究表明 Lactobacillusparacasei, Bifidobacteriumbreve 和 Lactobacillus rhamnosus 可以减少肥胖 大鼠的肝脏脂肪变, 不仅如此, 这些菌种还可以降低 LPS, TNF- $\alpha$, IL-6 等炎症因子的表达 ${ }^{[85]}$. 总而言之, 了 解肠道菌群在肝脏疾病中的调控作用具有重要意义.

\section{5 结论}

NAFLD 发生发展是多种因素共同作用的结果, 肝脏脂肪堆积是其核心表型，该病常伴随胰岛素抵 抗、糖脂代谢紊乱、细胞脂毒性损伤、炎症反应异常 等现象, 此外, 肠道菌群紊乱, 内毒素水平升高, 肠 道屏障通透性改变等可以调控这一疾病的发生发展. 这些表型往往相互联系、相互影响, 深入了解各个环 节的分子基础对于发展新型 NAFLD 诊断、分型、预 防以及靶向治疗策略和方法有重要意义.

\section{参考文献}

1 Choi S S, Diehl A M. Hepatic triglyceride synthesis and nonalcoholic fatty liver disease. Curr Opin Lipidol, 2008, 19: 295-300

2 Day C P, James O F. Steatohepatitis: a tale of two "hits"? Gastroenterology, 1998, 114: 842-845

3 Day C P, James O F. Hepatic steatosis: innocent bystander or guilty party? Hepatology, 1998, 27: 1463-1466

4 Merkel M, Eckel R H, Goldberg I J. Lipoprotein lipase: genetics, lipid uptake, and regulation. J Lipid Res, 2002, 43: 1997-2006

5 Arner P. Human fat cell lipolysis: biochemistry, regulation and clinical role. Best Pract Res Clin Endocrinol Metab, 2005, 19: 471-482

6 Pol A, Martin S, Fernandez M A, et al. Dynamic and regulated association of caveolin with lipid bodies: modulation of lipid body motility and function by a dominant negative mutant. Mol Biol Cell, 2004, 15: 99-110

7 Razani B, Combs T P, Wang X B, et al. Caveolin-1-deficient mice are lean, resistant to diet-induced obesity, and show 
hypertriglyceridemia with adipocyte abnormalities. J Biol Chem, 2002, 277: 8635-8647

8 Fernandez M A, Albor C, Ingelmo-Torres M, et al. Caveolin-1 is essential for liver regeneration. Science, 2006, 313: 1628-1632

9 Doege H, Baillie R A, Ortegon A M, et al. Targeted deletion of FATP5 reveals multiple functions in liver metabolism: alterations in hepatic lipid homeostasis. Gastroenterology, 2006, 130: 1245-1258

10 Goudriaan J R, Dahlmans V E, Teusink B, et al. CD36 deficiency increases insulin sensitivity in muscle, but induces insulin resistance in the liver in mice. J Lipid Res, 2003, 44: 2270-2277

11 Koonen D P, Jacobs R L, Febbraio M, et al. Increased hepatic CD36 expression contributes to dyslipidemia associated with diet-induced obesity. Diabetes, 2007, 56: 2863-1871

12 Luiken J J, Arumugam Y, Dyck D J, et al. Increased rates of fatty acid uptake and plasmalemmal fatty acid transporters in obese Zucker rats. J Biol Chem, 2001, 276: 40567-40573

13 Zhou J, Febbraio M, Wada T, et al. Hepatic fatty acid transporter Cd36 is a common target of LXR, PXR, and PPARgamma in promoting steatosis. Gastroenterology, 2008, 134: 556-567

14 Greco D, Kotronen A, Westerbacka J, et al. Gene expression in human NAFLD. Am J Physiol Gastrointest Liver Physiol, 2008, 294: G1281-1287

15 Tan N S, Shaw N S, Vinckenbosch N, et al. Selective cooperation between fatty acid binding proteins and peroxisome proliferator-activated receptors in regulating transcription. Mol Cell Biol, 2002, 22: 5114-5127

16 Helledie T, Antonius M, Sorensen R V, et al. Lipid-binding proteins modulate ligand-dependent trans-activation by peroxisome proliferator-activated receptors and localize to the nucleus as well as the cytoplasm. J Lipid Res, 2000, 41: 1740-1751

17 Wolfrum C, Borrmann C M, Borchers T, et al. Fatty acids and hypolipidemic drugs regulate peroxisome proliferator-activated receptors alpha - and gamma-mediated gene expression via liver fatty acid binding protein: a signaling path to the nucleus. Proc Natl Acad Sci USA, 2001, 98: 2323-2328

18 Xu A, Tso A W, Cheung B M, et al. Circulating adipocyte-fatty acid binding protein levels predict the development of the metabolic syndrome: a 5-year prospective study. Circulation, 2007, 115: 1537-1543

19 Tso A W, Xu A, Sham P C, et al. Serum adipocyte fatty acid binding protein as a new biomarker predicting the development of type 2 diabetes: a 10-year prospective study in a Chinese cohort. Diabetes Care, 2007, 30: 2667-2672

20 Shimano H, Horton J D, Shimomura I, et al. Isoform 1c of sterol regulatory element binding protein is less active than isoform 1a in livers of transgenic mice and in cultured cells. J Clin Invest, 1997, 99: 846-854

21 Ponugoti B, Kim D H, Xiao Z, et al. SIRT1 deacetylates and inhibits SREBP-1C activity in regulation of hepatic lipid metabolism. J Biol Chem, 2010, 285: 33959-33970

22 Liang G, Yang J, Horton J D, et al. Diminished hepatic response to fasting/refeeding and liver X receptor agonists in mice with selective deficiency of sterol regulatory element-binding protein-1c. J Biol Chem, 2002, 277: 9520-9528

23 Yellaturu C R, Deng X, Cagen L M, et al. Insulin enhances post-translational processing of nascent SREBP-1c by promoting its phosphorylation and association with COPII vesicles. J Biol Chem, 2009, 284: 7518-7532

24 Yecies J L, Zhang H H, Menon S, et al. Akt stimulates hepatic SREBP1c and lipogenesis through parallel mTORC1-dependent and independent pathways. Cell Metab, 2011, 14: 21-32

25 Owen J L, Zhang Y, Bae S H, et al. Insulin stimulation of SREBP-1c processing in transgenic rat hepatocytes requires p70 S6-kinase. Proc Natl Acad Sci USA, 2012, 109: 16184-16189

26 Bakan I, Laplante M. Connecting mTORC1 signaling to SREBP-1 activation. Curr Opin Lipidol, 2012, 23: 226-234

27 Toriumi K, Horikoshi Y, Yoshiyuki Osamura R, et al. Carbon tetrachloride-induced hepatic injury through formation of oxidized diacylglycerol and activation of the PKC/NF-kappaB pathway. Lab Invest, 2013, 93: 218-229

28 Zhang $\mathrm{H}$, Li Y, Hu J, et al. Effect of Creosote Bush-Derived NDGA on Expression of Genes Involved in Lipid Metabolism in Liver of High-Fructose Fed Rats: Relevance to NDGA Amelioration of Hypertriglyceridemia and Hepatic Steatosis. PLoS One, 2015, 10: e0138203

29 Kursawe R, Caprio S, Giannini C, et al. Decreased transcription of ChREBP-alpha/beta isoforms in abdominal subcutaneous adipose tissue of obese adolescents with prediabetes or early type 2 diabetes: associations with insulin resistance and hyperglycemia. Diabetes, 2013, 62: 837-844

30 Ma L, Tsatsos N G, Towle H C. Direct role of ChREBP.Mlx in regulating hepatic glucose-responsive genes. J Biol Chem, 2005, 280: 12019-12027

31 Ma L, Robinson L N, Towle H C. ChREBP*Mlx is the principal mediator of glucose-induced gene expression in the liver. J Biol Chem, 
2006, 281: 28721-28730

32 Girard J, Ferre P, Foufelle F. Mechanisms by which carbohydrates regulate expression of genes for glycolytic and lipogenic enzymes. Annu Rev Nutr, 1997, 17: 325-352

33 Iizuka K, Bruick R K, Liang G, et al. Deficiency of carbohydrate response element-binding protein (ChREBP) reduces lipogenesis as well as glycolysis. Proc Natl Acad Sci USA, 2004, 101: 7281-7286

34 Dentin R, Benhamed F, Hainault I, et al. Liver-specific inhibition of ChREBP improves hepatic steatosis and insulin resistance in ob/ob mice. Diabetes, 2006, 55: 2159-2170

35 Benhamed F, Denechaud P D, Lemoine M, et al. The lipogenic transcription factor ChREBP dissociates hepatic steatosis from insulin resistance in mice and humans. J Clin Invest, 2012, 122: 2176-2194

36 Beaven S W, Matveyenko A, Wroblewski K, et al. Reciprocal regulation of hepatic and adipose lipogenesis by liver X receptors in obesity and insulin resistance. Cell Metab, 2013, 18: 106-117

37 Kalaany N Y, Gauthier K C, Zavacki A M, et al. LXRs regulate the balance between fat storage and oxidation. Cell Metab, 2005, 1: 231-244

38 Yoshikawa T, Shimano H, Amemiya-Kudo M, et al. Identification of liver X receptor-retinoid X receptor as an activator of the sterol regulatory element-binding protein 1c gene promoter. Mol Cell Biol, 2001, 21: 2991-3000

39 Chen G, Liang G, Ou J , et al. Central role for liver X receptor in insulin-mediated activation of Srebp-1c transcription and stimulation of fatty acid synthesis in liver. Proc Natl Acad Sci USA, 2004, 101: 11245-11250

40 Cha J Y, Repa J J. The liver X receptor (LXR) and hepatic lipogenesis. The carbohydrate-response element-binding protein is a target gene of LXR. J Biol Chem, 2007, 282: 743-751

41 Mitro N, Mak P A, Vargas L, et al. The nuclear receptor LXR is a glucose sensor. Nature, 2007, 445: 219-223

42 Denechaud P D, Bossard P, Lobaccaro J M, et al. ChREBP, but not LXRs, is required for the induction of glucose-regulated genes in mouse liver. J Clin Invest, 2008, 118: 956-964

43 Joseph S B, Laffitte B A, Patel P H, et al. Direct and indirect mechanisms for regulation of fatty acid synthase gene expression by liver X receptors. J Biol Chem, 2002, 277: 11019-11025

44 Zhang Y, Castellani L W, Sinal C J, et al. Peroxisome proliferator-activated receptor-gamma coactivator 1alpha (PGC-1alpha) regulates triglyceride metabolism by activation of the nuclear receptor FXR. Genes Dev, 2004, 18: 157-169

45 Watanabe M, Houten S M, Wang L, et al. Bile acids lower triglyceride levels via a pathway involving FXR, SHP, and SREBP-1c. J Clin Invest, 2004, 113: 1408-1418

46 Sinal C J, Tohkin M, Miyata M, et al. Targeted disruption of the nuclear receptor FXR/BAR impairs bile acid and lipid homeostasis. Cell, 2000, 102: 731-744

$47 \mathrm{Xu} \mathrm{J}$, Xiao G, Trujillo C, et al. Peroxisome proliferator-activated receptor alpha (PPARalpha) influences substrate utilization for hepatic glucose production. J Biol Chem, 2002, 277: 50237-50244

48 Kersten S. Peroxisome proliferator activated receptors and obesity. Eur J Pharmacol, 2002, 440: 223-234

49 Pawlak M, Lefebvre P, Staels B. Molecular mechanism of PPARalpha action and its impact on lipid metabolism, inflammation and fibrosis in non-alcoholic fatty liver disease. J Hepatol, 2015, 62: 720-733

50 Hotamisligil G S. Endoplasmic reticulum stress and the inflammatory basis of metabolic disease. Cell, 2010, 140: 900-917

51 Finn P F, Dice J F. Proteolytic and lipolytic responses to starvation. Nutrition, 2006, 22: 830-844

52 Shibata M, Yoshimura K, Furuya N, et al. The MAP1-LC3 conjugation system is involved in lipid droplet formation. Biochem Biophys Res Commun, 2009, 382: 419-423

53 Li S, Brown M S, Goldstein J L. Bifurcation of insulin signaling pathway in rat liver: mTORC1 required for stimulation of lipogenesis, but not inhibition of gluconeogenesis. Proc Natl Acad Sci U S A, 2010, 107: 3441-3446

54 Rautou P E, Mansouri A, Lebrec D, et al. Autophagy in liver diseases. J Hepatol, 2010, 53: 1123-34

55 Neuschwander-Tetri B A. Hepatic lipotoxicity and the pathogenesis of nonalcoholic steatohepatitis: the central role of nontriglyceride fatty acid metabolites. Hepatology, 2010, 52: 774-788

56 McGarry J D, Brown N F. The mitochondrial carnitine palmitoyltransferase system. From concept to molecular analysis. Eur J Biochem, 1997, 244: 1-14

57 Dietrich P, Hellerbrand C. Non-alcoholic fatty liver disease, obesity and the metabolic syndrome. Best Pract Res Clin Gastroenterol, 2014, 28: 637-653

58 Sparks J D, Dong H H. FoxO1 and hepatic lipid metabolism. Curr Opin Lipidol, 2009, 20: 217-226 
59 Francque S, Verrijken A, Caron S, et al. PPARalpha gene expression correlates with severity and histological treatment response in patients with non-alcoholic steatohepatitis. J Hepatol, 2015, 63: 164-173

60 Teodoro J S, Rolo A P, Palmeira C M. Hepatic FXR: key regulator of whole-body energy metabolism. Trends Endocrinol Metab, 2011, 22: 458-466

61 Puri P, Baillie R A, Wiest M M, et al. A lipidomic analysis of nonalcoholic fatty liver disease. Hepatology, 2007, 46: 1081-1090

62 Chiu H C, Kovacs A, Blanton R M , et al. Transgenic expression of fatty acid transport protein 1 in the heart causes lipotoxic cardiomyopathy. Circ Res, 2005, 96: 225-233

63 Newberry E P, Xie Y, Kennedy S, et al. Decreased hepatic triglyceride accumulation and altered fatty acid uptake in mice with deletion of the liver fatty acid-binding protein gene. J Biol Chem, 2003, 278: 51664-51672

64 Spann N J, Kang S, Li A C, et al. Coordinate transcriptional repression of liver fatty acid-binding protein and microsomal triglyceride transfer protein blocks hepatic very low density lipoprotein secretion without hepatosteatosis. J Biol Chem, 2006, 281: 33066-33077

65 Nolan C J, Larter C Z. Lipotoxicity: why do saturated fatty acids cause and monounsaturates protect against it? J Gastroenterol Hepatol, 2009, 24: 703-706

66 Fessler M B, Rudel L L, Brown J M. Toll-like receptor signaling links dietary fatty acids to the metabolic syndrome. Curr Opin Lipidol, 2009, 20: 379-385

67 Li Z, Berk M, McIntyre T M, et al. The lysosomal-mitochondrial axis in free fatty acid-induced hepatic lipotoxicity. Hepatology, 2008, 47: 1495-1503

68 Erbay E, Babaev V R, Mayers J R, et al. Reducing endoplasmic reticulum stress through a macrophage lipid chaperone alleviates atherosclerosis. Nat Med, 2009, 15: 1383-1391

69 Minehira K, Young S G, Villanueva C J, et al. Blocking VLDL secretion causes hepatic steatosis but does not affect peripheral lipid stores or insulin sensitivity in mice. J Lipid Res, 2008, 49: 2038-2044

70 Novgorodov S A, Szulc Z M, Luberto C, et al. Positively charged ceramide is a potent inducer of mitochondrial permeabilization. J Biol Chem, 2005, 280: 16096-16105

71 Han M S, Park S Y, Shinzawa K, et al. Lysophosphatidylcholine as a death effector in the lipoapoptosis of hepatocytes. J Lipid Res, 2008, 49: 84-97

72 Wei Y, Wang D, Topczewski F, et al. Saturated fatty acids induce endoplasmic reticulum stress and apoptosis independently of ceramide in liver cells. Am J Physiol Endocrinol Metab, 2006, 291: E275-81

73 Listenberger L L, Ory D S, Schaffer J E. Palmitate-induced apoptosis can occur through a ceramide-independent pathway. J Biol Chem, 2001, 276: 14890-14895

74 Lewis J R, Mohanty S R. Nonalcoholic fatty liver disease: a review and update. Dig Dis Sci, 2010, 55: 560-578

75 Zhan Y T, An W. Roles of liver innate immune cells in nonalcoholic fatty liver disease. World J Gastroenterol, 2010, 16: 4652-4660

76 Sachdev M S, Riely C A, Madan A K. Nonalcoholic fatty liver disease of obesity. Obes Surg, 2006, 16: 1412-1419

77 Xu P, Zhang X G, Li Y M, et al. Research on the protection effect of pioglitazone for non-alcoholic fatty liver disease (NAFLD) in rats. J Zhejiang Univ Sci B, 2006, 7: 627-633

78 Samuel V T, Liu Z X, Qu X, et al. Mechanism of hepatic insulin resistance in non-alcoholic fatty liver disease. J Biol Chem, 2004, 279: 32345-32353

79 Kumashiro N, Erion D M, Zhang D, et al. Cellular mechanism of insulin resistance in nonalcoholic fatty liver disease. Proc Natl Acad Sci USA, 2011, 108: 16381-16385

80 Adams D H, Eksteen B, Curbishley S M. Immunology of the gut and liver: a love/hate relationship. Gut, 2008, 57: 838-848

81 Raman M, Ahmed I, Gillevet P M, et al. Fecal microbiome and volatile organic compound metabolome in obese humans with nonalcoholic fatty liver disease. Clin Gastroenterol Hepatol, 2013, 11: 868-75 e1-3

82 Mouzaki M, Comelli E M, Arendt B M, et al. Intestinal microbiota in patients with nonalcoholic fatty liver disease. Hepatology, 2013, 58: 120-127

83 Zhu L, Baker S S, Gill C, et al. Characterization of gut microbiomes in nonalcoholic steatohepatitis (NASH) patients: a connection between endogenous alcohol and NASH. Hepatology, 2013, 57: 601-609

84 Xu R Y, Wan Y P, Fang Q Y, et al. Supplementation with probiotics modifies gut flora and attenuates liver fat accumulation in rat nonalcoholic fatty liver disease model. J Clin Biochem Nutr, 2012, 50: 72-77

85 Wang J, Tang H, Zhang C, et al. Modulation of gut microbiota during probiotic-mediated attenuation of metabolic syndrome in high fat diet-fed mice. ISME J, 2015, 9: 1-15 


\title{
Hepatic lipid metabolism and non-alcoholic fatty liver disease
}

\author{
QU YuLan \& HAN ZeGuang
}

Shanghai center for systems biomedicine, Shanghaijiaotong university, Shanghai 200240, China

The prevalence of non-alcoholic fatty liver disease (NAFLD) along with the metabolic diseases such as obesity, type 2 diabetes and metabolic syndrome keeps rising worldwide. Unfortunately, our understanding of what causes NAFLD at the molecular level remains mostly speculative. Insulin resistance, lipotoxicity and inflammation may be involved in the pathogenesis of this disease. In the current review, we discuss the role of disturbed lipid metabolism in the development of NAFLD, and overview other altered factors that may participate in this process, including lipotoxicity, insulin resistance as well as intestinal flora disorder. Our aims are to provide new insights into prevention and potential therapeutic strategies for NAFLD.

\section{NAFLD, lipid metabolism}

doi: 10.1360/N052017-00103 